\title{
Kodama-Schwarzschild versus Gaussian Normal Coordinates Picture of Thin Shells
}

\author{
Mikhail Z. Iofa \\ Skobeltsyn Institute of Nuclear Physics, Moscow State University, Moscow 119991, Russia \\ Correspondence should be addressed to Mikhail Z. Iofa; iofa@theory.sinp.msu.ru
}

Received 6 September 2016; Accepted 2 November 2016

Academic Editor: S. Habib Mazharimousavi

Copyright (C) 2016 Mikhail Z. Iofa. This is an open access article distributed under the Creative Commons Attribution License, which permits unrestricted use, distribution, and reproduction in any medium, provided the original work is properly cited. The publication of this article was funded by SCOAP $^{3}$

Geometry of the spacetime with a spherical shell embedded in it is studied in two coordinate systems: Kodama-Schwarzschild coordinates and Gaussian normal coordinates. We find explicit coordinate transformation between the Kodama-Schwarzschild and Gaussian normal coordinate systems. We show that projections of the metrics on the surface swept by the shell in the 4D spacetime in both cases are identical. In the general case of time-dependent metrics we calculate extrinsic curvatures of the shell in both coordinate systems and show that the results are identical. Applications to the Israel junction conditions are discussed.

\section{Introduction}

Thin shells provide a useful tool to study dynamics of collapsing body eventually forming black hole and Hawking radiation from the black hole $[1,2]$.

Dynamics of domain walls was studied by Israel [3], Poisson [4], Ipser and Sikivie [5], Berezin et al. [6], Blau et al. [7], Chowdhury [8], Gladush [9], Kraus and Wilczek [10], and many other authors.

There are two natural settings to study geometry of the spacetime with a spherical shell: that based on KodamaSchwarzschild coordinates and that employing the Gaussian normal coordinate system.

Kodama found that in any (possibly time-dependent) spherically symmetric spacetime there exists a conserved vector which is timelike in the exterior of the shell [11]. Although the Kodama vector does not reduce to the Killing vector even in the static spacetime, it can be used to define a preferred "time coordinate" and to construct a geometrically preferred coordinate system for a spherically symmetric spacetime [11-15]. Because the Kodama vector is orthogonal to $d r$, one can construct the time coordinate $t$ so that $d r$ is orthogonal to $d t$ [15]. Using the Schwarzschild radial coordinate $r$, one arrives at the diagonal, time-dependent spherically symmetric metric which in this parametrization in the $(r, t)$ sector has the metric components $g_{r r}=(1-$ $2 m(t, r) / r)^{-1}$ and $g_{t t}=b^{2}(r, t)(1-2 m(t, r) / r)$.
The Gaussian normal coordinate system in the neighborhood of the shell $[7,16]$ is constructed by using a family of nonintersecting geodesics orthogonal to the surface $\Sigma$ swept by the shell. Coordinates of a point outside of the shell are introduced as the geodesic distance from the point to the shell along the geodesic orthogonal to the surface $\Sigma$ and coordinates of the intersection point of the geodesic with $\Sigma$.

The aim of the present paper is to study connection between two approaches. We find explicit coordinate transformation between the Kodama -Schwarzschild and Gaussian normal coordinate systems. We show that projections of the metrics on the surface swept by the shell in the $4 \mathrm{D}$ spacetime in both cases are identical. In the general case of time-dependent metrics we calculate extrinsic curvatures of the shell in both coordinate systems and show that the results are identical. Applications of the above results to the Israel junction conditions are discussed.

\section{Kodama-Schwarzschild Coordinates}

The $(2+1)$ dimensional hypersurface $\Sigma$ swept by a spherically symmetric shell divides $4 \mathrm{D}$ spacetime in two regions $V^{ \pm}$. Any spherically symmetric metric in $D=1+3$ spacetime has the general form 


$$
\begin{aligned}
d s^{2} & =g_{a, b}(x) d x^{a} d x^{b} \\
& ={ }^{(2)} g_{i, j}(x) d x^{i} d x^{j}+r^{2}(x) d \Omega^{2} .
\end{aligned}
$$

Here $x^{a}=\left(x^{i}, x^{\alpha}\right)$, where $x^{i}$ are coordinates in the base space and $\theta$ and $\varphi$ are coordinates on the spherically symmetric fibers. For any spherically symmetric spacetime it is possible to introduce a vector $k^{a}=\varepsilon_{\perp}^{a b} \nabla_{b} r$ (Kodama vector) $[11-15,17]$, which lies in the radial-temporal plane, where $\varepsilon_{\perp}^{a b}$

$$
\varepsilon_{\perp}^{a b}=\left(\begin{array}{cc}
\varepsilon_{\perp}^{i j} & 0 \\
0 & 0
\end{array}\right) .
$$

By construction Kodama vector $k$ is orthogonal to $\nabla_{a} r$. Choosing the time coordinate $t$ so that $\partial_{t} \sim k$ [15], one obtains the metric in the diagonal form because $k$ and $d t$ are orthogonal to $d r$. In the parametrization through the time coordinate $t$ and Schwarzschild radial coordinate $r$ the metric can be expressed as

$$
d s_{ \pm}^{2}=-b_{ \pm}^{2}\left(r, t_{ \pm}\right) f_{ \pm}\left(r, t_{ \pm}\right) d t_{ \pm}^{2}+\frac{d r^{2}}{f_{ \pm}\left(r, t_{ \pm}\right)}+r^{2} d \Omega^{2},
$$

where $f_{ \pm}\left(r, t_{ \pm}\right)=\left(1-2 m_{ \pm}\left(r, t_{ \pm}\right) / r\right)$. In this parametrization $m(r, t)$ is interpreted as the quasi-local mass (Misner-SharpHernandez mass) $[18,19]$. Note that in this parametrization the metric is diagonal.

Position of the surface is defined by parametric equations $r=R(\tau), t_{ \pm}=T_{ \pm}(\tau)$. The metrics induced on the shell from the regions $V^{ \pm}$are

$$
\begin{aligned}
d s_{ \pm}^{2}= & -b_{ \pm}^{2}\left(R, T_{ \pm}\right) f_{ \pm}\left(R, T_{ \pm}\right) d T_{ \pm}^{2}+\frac{d R^{2}}{f_{ \pm}\left(R, T_{ \pm}\right)} \\
& +R^{2} d \Omega^{2}
\end{aligned}
$$

From the requirement that the metrics induced on $\Sigma$ from both regions $V^{ \pm}$coincide (first Israel condition) it follows that

$$
\begin{aligned}
& -b_{+}^{2}\left(R, T_{+}\right) f_{+}\left(R, T_{+}\right) \dot{T}_{+}^{2}+f_{+}^{-1}\left(R, T_{+}\right) \dot{R}^{2} \\
& =-b_{-}^{2}\left(R, T_{-}\right) f_{-}\left(R, T_{-}\right) \dot{T}_{-}^{2}+f_{-}^{-1}\left(R, T_{-}\right) \dot{R}^{2} .
\end{aligned}
$$

By choosing $\tau$ as the proper time on the surface, one obtains

$$
-b_{ \pm}^{2}\left(R, T_{ \pm}\right) f_{ \pm}\left(R, T_{ \pm}\right) \dot{T}_{ \pm}{ }^{2}+f_{ \pm}^{-1}\left(R, T_{ \pm}\right) \dot{R}^{2}=-1,
$$

and projection of the metric on $\Sigma$ is

$$
d s^{2}=-d \tau^{2}+R^{2}(\tau) d \Omega^{2} .
$$

\section{Gaussian Normal Coordinates}

Gaussian normal coordinate system in 4D spacetime in which a hypersurface swept by the spherical shell divides into two regions is introduced starting from a certain coordinate system $\widehat{x}^{\mu}$ with a metric $\widehat{g}_{\mu \nu}(\widehat{x})$. The surface $\Sigma$ is parametrized by coordinates $x^{i}=(\xi, \theta, \varphi)$ Consider a neighborhood of $\Sigma$ with a system of geodesics orthogonal to $\Sigma$. The neighborhood is chosen so that the geodesics do not intersect; that is, any point in the neighborhood is located on one and only one geodesic. Let us consider a point in the neighborhood of $\Sigma$ with the geodesic orthogonal to $\Sigma$ which goes through this point. The new coordinate system $x^{\mu}$ is introduced in the following way. Three coordinates of the point coincide with the coordinates $x^{i}$ of the point of intersection of the geodesic with $\Sigma$. The fourth coordinate of a point is equal to the proper geodesic distance along the geodesic from the point to $\Sigma$. The proper length along the geodesic is

$$
\eta=\int_{0}^{\eta} d \eta^{\prime} \sqrt{g_{\mu \nu}\left(x^{\mu}\right) \frac{d x^{\mu}}{d \eta^{\prime}} \frac{d x^{\nu}}{d \eta^{\prime}}}
$$

where $\eta$ is the affine parameter along the geodesic. Expression (8) is invariant under the coordinate transformations with Jacobian equal to unity, and we can rewrite (8) through the new coordinates $x^{\mu}$ and the metric $g_{\mu \nu}(x)$. Taking the derivative over $\eta$ from both sides of (8) over $\eta$, one has

$$
g_{\mu \nu}(x) \frac{\partial x^{\mu}\left(\eta, x^{i}\right)}{\partial \eta} \frac{\partial x^{\nu}\left(\eta, x^{i}\right)}{\partial \eta}=1
$$

or $g_{\eta \eta}\left(\eta, x^{i}\right)=1$. Orthogonality condition of the tangent vector to geodesic to the tangent surface to $\Sigma$ is

$$
\left.g_{\mu \nu}(x) \frac{\partial x^{\mu}\left(\eta, x^{i}\right)}{\partial \eta} \frac{\partial x^{\nu}\left(\eta, x^{i}\right)}{\partial x^{i}}\right|_{\eta=0}=0
$$

or $g_{\eta i}\left(0, x^{i}\right)=0$. The tangent vector $\partial x^{\mu}\left(\eta, x^{i}\right) /\left.\partial \eta\right|_{\eta=0}$ is orthogonal to $\Sigma$ and the vector $\partial x^{\nu}\left(0, x^{i}\right) / \partial x^{i}$ is in the plane tangent to $\Sigma$.

The metrics in $V^{ \pm}$are (below, to simplify formulas, we omit the subscript \pm everywhere, where it does not lead to confusion)

$$
\begin{aligned}
d s_{ \pm}^{2} & =d \eta^{2}-p^{2}(\eta, \xi) d \xi^{2}+2 q(\eta, \xi) d \tau d \eta \\
& +\left.\rho^{2}(\eta, \xi) d \Omega^{2}\right|_{ \pm} .
\end{aligned}
$$

Because of condition (10), on the surface $\Sigma$ the interval reduces to

$$
d s^{2}=-p^{2}(0, \xi) d \xi^{2}+\rho^{2}(0, \tau) d \Omega^{2} .
$$

On the surface $\Sigma$ reparametrization of $\tau$ allows setting $p^{2}(0, \xi)=1$, which is assumed in the following. It is seen that one can identify $\tau$ with $\xi$ and $R(\tau)$ with $\rho(0, \xi)$. In the following we use the variable $\tau$.

\section{Transformation between the Coordinate Systems}

Coordinate transformation $t_{ \pm}=t_{ \pm}(\eta, \tau)$ and $r_{ \pm}=r(\eta, \tau)$ from Kodama-Schwarzschild coordinates $x^{\mu}=(t, r, \theta, \varphi)$ to Gaussian normal coordinates $\widehat{x}^{\mu}=(\eta, \tau, \theta, \varphi)$ yields the 
following relations between the components of the metrics (6) and (11):

$$
\begin{aligned}
-b^{2} f \dot{t}^{2}+f^{-1} \dot{r}^{2} & =-p^{2}, \\
-b^{2} f t^{\prime 2}+f^{-1} r^{\prime 2} & =1, \\
-b^{2} f \dot{t} t^{\prime}+f^{-1} \dot{r} r^{\prime} & =q,
\end{aligned}
$$

where prime and dot denote derivatives over $\eta$ and $\tau$. On the surface $\Sigma$ transformations (13)-(15) are of the same form with the substitution $\dot{t} \rightarrow \dot{T}, \dot{r} \rightarrow \dot{R}$ and $q=0, p=1$.

It is straightforward to obtain solution of systems (13)(15) in the spacetime regions $V^{ \pm}$as $\dot{t}=\dot{t}(p, q, \dot{r}), t^{\prime}=$ $t^{\prime}(p, q, \dot{r})$, and $r^{\prime}=r^{\prime}(p, q, \dot{r})$. Instead of writing this cumbersome and not instructive general solution, we consider the restriction of the transformation to the surface $\Sigma$ which we use as follows:

$$
\begin{aligned}
\left.\dot{t}^{2}\right|_{\Sigma} & =\dot{T}^{2}=\frac{f(T, R)+\dot{R}^{2}}{b^{2}(T, R) f^{2}(T, R)}, \\
\left.t^{\prime 2}\right|_{\Sigma} & =\frac{\dot{R}^{2}}{b^{2}(T, R) f^{2}(T, R)}, \\
\left.r^{\prime 2}\right|_{\Sigma} & =f(T, R)+\dot{R}^{2} .
\end{aligned}
$$

Because $\Sigma$ is orientable, on $\Sigma$ a normal vector can be defined. In Kodama-Schwarzschild coordinates tangent, $u_{\mu}$, and normal, $n_{\mu}$, vectors to the surface $\Sigma$ at either side of the surface are

$$
\begin{aligned}
& u_{ \pm}^{\mu}=\left(\dot{T}_{ \pm}, \dot{R}, 0,0\right), \quad u^{2}=1, \\
& n_{\mu \pm}= \pm N\left(-\dot{R}, \dot{T}_{ \pm}, 0,0\right), \quad u^{\mu} n_{\mu}=0, \\
& n_{ \pm}^{\mu}= \pm N\left(\frac{\dot{R}}{b_{ \pm}^{2} f_{ \pm}}, \dot{T}_{ \pm} f_{ \pm}, 0,0\right) .
\end{aligned}
$$

Normalizing $n^{2}$ to unity, we obtain

$$
n^{2}=N^{2}\left(-\frac{\dot{R}^{2}}{b^{2} f}+\dot{T}^{2} f\right)=\frac{N^{2}}{b^{2}}=1 .
$$

Transformations of the components of the tangent vector from Kodama-Schwarzschild coordinates $(t, r)$ to Gaussian coordinates $(\tau, \eta)$ are

$$
\begin{gathered}
\widehat{u}_{\eta}^{ \pm}=\left.u_{\mu}^{ \pm} \frac{\partial x_{ \pm}^{\mu}}{\partial \eta}\right|_{\Sigma}=-b^{2} f \dot{T} t^{\prime}+\left.\frac{\dot{R} r^{\prime}}{f}\right|_{\Sigma} ^{ \pm}=0, \\
\left.\widehat{u}_{\tau}^{ \pm}\right|_{\Sigma}=\left.u_{\mu}^{ \pm} \frac{\partial x_{ \pm}^{\mu}}{\partial \tau}\right|_{\Sigma}=-b^{2} f \dot{T} \dot{t}+\left.\frac{\dot{R} \dot{r}}{f}\right|_{\Sigma} ^{ \pm}=-1 .
\end{gathered}
$$

The corresponding transformations of the components of the normal vector are

$$
\begin{aligned}
& \widehat{n}_{\tau}^{ \pm}=\left.n_{\mu}^{ \pm} \frac{\partial x_{ \pm}^{\mu}}{\partial \tau}\right|_{\Sigma}=-\dot{R} \dot{t}+\left.\dot{T} \dot{r}\right|_{\Sigma} ^{ \pm}=0, \\
& \widehat{n}_{\eta}^{ \pm}=\left.n_{\mu}^{ \pm} \frac{\partial x_{ \pm}^{\mu}}{\partial \eta}\right|_{\Sigma}=\left.N\left(-\dot{R} t^{\prime}+\dot{T} r^{\prime}\right)\right|_{\Sigma} ^{ \pm}= \pm 1 .
\end{aligned}
$$

In (22) and (23) we used expressions (16), where all the square roots for $\dot{T},\left.t^{\prime}\right|_{\Sigma}$, and $r_{\Sigma}^{\prime}$ are taken with the same signs. The upper sign in (23) corresponds to the square roots taken with the sign $(+)$.

Next, we consider another method to construct the explicit form of the coordinate transformation from KodamaSchwarzschild coordinates to Gaussian normal coordinates. The problem can be solved in principle by solving the geodesic equations:

$$
\frac{d^{2} x^{\mu}}{d \lambda^{2}}+\Gamma_{\nu \lambda}^{\mu} \frac{d x^{\nu}}{d \lambda} \frac{d x^{\lambda}}{d \lambda}=0
$$

In the general case with metric (3) depending on $t$ the system of nonlinear differential equations is not tractable. Explicit relations can be obtained in the case of metric (3) with the components independent of $t$ (in this case it is possible to set $b(r)=1$ : introducing new variable $\rho$ by the relation $d \rho=d r / b(r)$ and denoting $k(\rho)=b^{2} f(r(\rho))$, we obtain the metric (3) with $b=1$.). In this case the geodesic equations in Kodama -Schwarzschild coordinates are

$$
\begin{aligned}
& \frac{d^{2} t}{d \lambda^{2}}+\frac{f_{, r}}{f} \frac{d t}{d \lambda} \frac{d r}{d \lambda}=0 \\
& \frac{d^{2} r}{d \lambda^{2}}+\frac{f_{, r} f}{2}\left(\frac{d t}{d \lambda}\right)^{2}-\frac{f_{, r}}{2 f}\left(\frac{d r}{d \lambda}\right)^{2}+f r\left(\frac{d \theta}{d \lambda}\right)^{2} \\
& \quad+f r \sin ^{2} \theta\left(\frac{d \varphi}{d \lambda}\right)^{2}=0, \\
& \frac{d^{2} \theta}{d \lambda^{2}}=0=\frac{d^{2} \varphi}{d \lambda^{2}},
\end{aligned}
$$

where $\lambda$ is affine parameter. The first integrals of the system of equations are

$$
\begin{aligned}
& \frac{d t}{d \lambda}=\frac{C_{t}(\tau)}{f(r)}, \\
& \frac{d r}{d \lambda}= \pm\left(f(r)+C_{t}^{2}(\tau)-C_{\theta} f(r) r^{2}\right)^{1 / 2}, \\
& \frac{d \theta}{d \lambda}=C_{\theta}, \\
& \frac{d \varphi}{d \lambda}=C_{\varphi} .
\end{aligned}
$$

Here $r=r(\lambda, \tau), t=t(\lambda, \tau)$. To maintain spherical symmetry, we take $C_{\theta}=C_{\varphi}=0$. By construction the vector $l^{\mu}(\lambda$, $\tau)=(\partial t / \partial \lambda, \partial r / \partial \lambda, 0,0)$ is tangent to the geodesic. Let us consider the geodesics orthogonal to $\Sigma$. In this case the affine parameter $\lambda$ can be identified with the parameter $\eta$. From (26) it follows that the vector $l^{\mu}$ is normalized to unity. At the surface $\Sigma$ the vector $l^{\mu}$ up to the sign coincides with the normal vector (19). Thus, at the surface $\Sigma$ we have $C_{t}^{2}=\dot{R}^{2}$. For $l^{\mu}(\lambda, \tau)$ we obtain

$$
l^{\mu}= \pm\left(\frac{\dot{R}}{f(r)}, \sqrt{f(r)+\dot{R}^{2}}, 0,0\right) .
$$

On the surface $\Sigma$ solution (27) coincides with formulas (16). 
In Kodama-Schwarzschild parametrization the variables $\tau$ and $\eta$ have a clear geometrical meaning: at the $(t, r)$ plane $\tau$ varies along the trajectory of the shell $(R(\tau), T(\tau))$, and $\eta$ varies along the geodesics orthogonal to the surface swept by the shell.

\section{Extrinsic Curvature}

The extrinsic curvatures at either side of $\Sigma$ are

$$
K_{i j}^{ \pm}=\left(h_{\mu}^{\lambda} n_{v ; \lambda} \frac{d x^{\mu}}{d x^{i}} \frac{d x^{\nu}}{d x^{j}}\right)_{\Sigma}^{ \pm}
$$

where $x^{i}=(\tau, \theta, \varphi)$ are coordinates on $\Sigma, h_{\mu \nu}=g_{\mu \nu}-$ $n_{\mu} n_{v}$, and (;) denote covariant derivative with respect to $g_{\mu \nu}^{ \pm}$. In Kodama-Schwarzschild parametrization $\left.x^{\mu}\right|_{\Sigma}=$ $(T, R, \theta, \varphi)$. In Kodama-Schwarzschild parametrization the nonzero components of the extrinsic curvature are

$$
\begin{aligned}
K_{\tau \tau}^{ \pm} & =\left.h_{\mu}^{\lambda} n_{v ; \lambda} u^{\mu} u^{\nu}\right|^{ \pm}=\left.n_{v ; \lambda} u^{\lambda} u^{\nu}\right|^{ \pm}, \\
K_{\theta \theta}^{ \pm} & =\left.h_{\mu}^{\lambda} n_{v ; \lambda} \frac{d x^{\mu}}{d \theta} \frac{d x^{\nu}}{d \theta}\right|^{ \pm}=\left.h_{\theta}^{\lambda} n_{\theta ; \lambda}\right|^{ \pm}=\left.n_{\theta ; \theta}^{ \pm}\right|^{ \pm} \\
& =-\left.\Gamma_{\theta \theta}^{R} n_{R}\right|^{ \pm}, \\
K_{\varphi \varphi}^{ \pm} & =n_{\varphi ; \varphi}^{ \pm}=-\left.\Gamma_{\varphi \varphi}^{R} n_{R}\right|^{ \pm} .
\end{aligned}
$$

Using the identity $n_{\mu ;} u^{\mu} u^{\nu}=-n_{\mu} u_{; \gamma}^{\mu} u^{\nu}$, we have $K_{\tau \tau}=$ $-u^{v} u_{; \nu}^{\mu} n_{\mu}$. From the identity $u_{v} u^{v}=1$ it follows that $u^{\mu} u_{\mu ; \nu} v^{v}=$ 0 or $u_{T} u_{; \nu}^{T} u^{\nu}+u_{R} u_{; \nu}^{R} u^{\nu}=0$. Direct calculation yields

$$
u_{; \nu}^{R} u^{\nu}=\ddot{R}+\dot{T}^{2} \Gamma_{T T}^{R}+\dot{R}^{2} \Gamma_{R R}^{R}+2 \dot{T} \dot{R} \Gamma_{R T}^{R} .
$$

$K_{\tau \tau}$ is expressed as

$$
\begin{aligned}
K_{\tau \tau} & =-u^{\nu} u_{; \nu}^{\mu} n_{\mu}=u_{; \nu}^{R} u^{\nu}\left(n_{T} \frac{u_{R}}{u_{T}}-n_{R}\right) \\
& =-\frac{u_{; \nu}^{R} u^{\nu}}{\dot{T} b(R, T) f(R, T)} .
\end{aligned}
$$

Using the expressions of Section 4 for $u_{\mu}$ and $n_{\mu}$ with the upper signs, we obtain the extrinsic curvature in KodamaSchwarzschild coordinates

$$
\begin{aligned}
K_{\tau \tau}^{ \pm} & =-\frac{1}{\sqrt{\dot{R}^{2}+f(R, T)}}\left(\ddot{R}+\frac{f_{, r}(R, T)}{2}\right. \\
& \left.+\left(f(R, T)+\dot{R}^{2}\right) \frac{b_{, r}(R, T)}{b(R, T)}-\dot{R} \dot{T} \frac{f_{, t}(R, T)}{f(R, T)}\right)\left.\right|^{ \pm}, \\
K_{\theta \theta}^{ \pm} & =\left.R \sqrt{\dot{R}^{2}+f(R, T)}\right|^{ \pm}, \\
K_{\varphi \varphi}^{ \pm} & =\left.R \sqrt{\dot{R}^{2}+f(R, T)} \sin ^{2} \theta\right|^{ \pm} .
\end{aligned}
$$

Here transforming from (31) to (32) we substituted

$$
\dot{T}_{ \pm} b\left(R, T_{ \pm}\right) f_{ \pm}\left(R, T_{ \pm}\right)=\left(f_{ \pm}\left(R, T_{ \pm}\right)+\dot{R}^{2}\right)^{1 / 2}
$$

which follows from (6).

In Gaussian normal coordinates the components of the extrinsic curvature are

$$
\begin{aligned}
& \widehat{K}_{\tau \tau}^{ \pm}=\widehat{n}_{\tau ; \tau}^{ \pm}=-\left.\Gamma_{\tau \tau}^{\eta} \widehat{n}_{\eta}\right|_{\Sigma} ^{ \pm}=-\left.\frac{1}{2}\left(p^{2}\right)_{, \eta}(\tau, \eta) \widehat{n}_{\eta}\right|_{\eta=0} ^{ \pm}, \\
& \widehat{K}_{\theta \theta}^{ \pm}=\widehat{n}_{\theta ; \theta}^{ \pm}=-\left.\Gamma_{\theta \theta}^{\eta} \widehat{n}_{\eta}\right|_{\eta=0} ^{ \pm}=\left.\frac{1}{2} r_{, \eta}^{2} \widehat{n}_{\eta}\right|_{\eta=0} ^{ \pm}, \\
& \widehat{K}_{\varphi \varphi}^{ \pm}=\widehat{n}_{\varphi ; \varphi}^{ \pm}=-\left.\Gamma_{\varphi \varphi}^{\eta} \widehat{n}_{\eta}\right|_{\eta=0} ^{ \pm}=\left.\frac{1}{2} r_{, \eta}^{2} \widehat{n}_{\eta} \sin ^{2} \theta\right|_{\eta=0} ^{ \pm} .
\end{aligned}
$$

In the general case of the functions $f(t, r)$ depending on $t$ calculation is straightforward but cumbersome. Below we perform calculation for the case of $f^{ \pm}(r)$ independent of $t$. Using solutions (27),

$$
\begin{aligned}
t^{\prime} & =\frac{\dot{R}}{f(r)}, \\
r^{\prime} & =\sqrt{f(r)+\dot{R}^{2}},
\end{aligned}
$$

we have

$$
\begin{aligned}
& \left.\dot{t}^{\prime}\right|_{\Sigma}=\frac{\ddot{R} f(R)-f_{, r}(R) \dot{R}^{2}}{f^{2}(R)}, \\
& \left.\dot{r}^{\prime}\right|_{\Sigma}=\frac{f_{, r}(R) \dot{R}+2 \dot{R} \ddot{R}}{2 \sqrt{f(R)+\dot{R}^{2}}} .
\end{aligned}
$$

Using (13), we obtain

$$
\begin{gathered}
\left.p_{, \eta}^{2}\right|_{\Sigma}=\left.\left(f \dot{t}^{2}-f^{-1} \dot{r}^{2}\right)_{; \eta}\right|_{\Sigma}=\left[f_{, r}(R) r^{\prime} \dot{T}^{2}\right. \\
\left.+2 f(R) \dot{T} \dot{t}^{\prime}+\frac{f_{, r}(R) r^{\prime} \dot{R}^{2}}{f^{2}(R)}-\frac{2 \dot{R} \dot{r}^{\prime}}{f(R)}\right]_{\Sigma} .
\end{gathered}
$$

Substituting expressions (38), we obtain

$$
\begin{aligned}
K_{\tau \tau}^{ \pm} & =-\frac{2 \ddot{R}+f_{, r}^{ \pm}(R)}{2 \sqrt{f^{ \pm}(R)+\dot{R}^{2}}}, \\
\widehat{K}_{\theta \theta}^{ \pm} & =R \sqrt{f^{ \pm}(R)+\dot{R}^{2}}, \\
\widehat{K}_{\varphi \varphi}^{ \pm} & =R \sqrt{f^{ \pm}(R)+\dot{R}^{2}} \sin ^{2} \theta .
\end{aligned}
$$

It is seen that extrinsic curvatures in both parametrizations coincide. 


\section{Israel Junction Conditions}

Next, we consider the Einstein equations and the Israel junction conditions. The energy-momentum tensor is taken in the form

$$
T_{\mu \nu}=\theta(\eta) S_{\mu \nu}^{+}+\theta(-\eta) S_{\mu \nu}^{-}+\delta(\eta) \delta_{\mu}^{i} \delta_{\nu}^{j} S_{i j}
$$

Because the values of the extrinsic curvatures at the opposite sides of $\Sigma$ are different, the derivative of the extrinsic curvature through the surface $\Sigma$ contains $\delta$-singularity. From the singular part of the $(i j)$ component of the Einstein equations projected on $\Sigma$,

$$
\begin{gathered}
{ }^{(3)} R_{j}^{i}-\delta_{j}^{i(3)} R-\left(K_{j}^{i}-\delta_{j}^{i} K\right)_{, \eta}-K K_{j}^{i} \\
+\left(K^{2}+K_{j}^{i} K_{i}^{j}\right) / 2=8 \pi G T_{j}^{i},
\end{gathered}
$$

follow the relations,

$$
\left[K_{j}^{i}\right]-\delta_{j}^{i}[K]=-8 \pi G S_{j}^{i},
$$

where $\left[K^{i}{ }_{j}\right]=K^{i+}{ }_{j}-K^{i-}{ }_{j}$ and $K=K^{i}{ }_{i}$. The $(\eta i)$ component of the Einstein equations,

$$
-K^{j}{ }_{i \mid j}+K_{, i}=8 \pi G T^{\eta}
$$

(vertical bar stands for covariant derivative with respect to metric (7)) yields

$$
K^{j \pm}{ }_{i \mid j}-K_{i}^{ \pm}=8 \pi G \theta( \pm \eta) S_{i}^{\eta \pm} .
$$

From the $(\eta \eta)$ component of the Einstein equations,

$$
-\frac{{ }^{(3)} R}{2}-\frac{K_{j}^{i} K_{i}^{j}}{2}-\frac{K^{2}}{2}=8 \pi G T_{\eta}^{\eta},
$$

it follows that

$$
-\frac{1}{2}\left(K_{j}^{i} K_{i}^{j}+K^{2}\right)^{ \pm}=8 \pi G \theta( \pm \eta) S_{\eta}^{\eta \pm} .
$$

Further restrictions on $S_{\mu \nu}^{ \pm}$follow from the conservation equations of the energy-momentum tensor [7].

Projections of the components of the bulk metric $g_{\mu \nu}$ defined as $\tilde{g}_{i j}=g_{\mu \nu} \partial x^{\mu} / \partial x^{i} \partial x^{\nu} /\left.\partial x^{j}\right|_{\Sigma}$ are

$$
\begin{aligned}
& \tilde{g}_{\tau \tau}=-1, \\
& \tilde{g}_{\theta \theta}=R^{2}(\tau), \\
& \tilde{g}_{\varphi \varphi}=R^{2}(\tau) \sin ^{2} \theta .
\end{aligned}
$$

Projections of the tangent vector $\tilde{u}_{i}=u_{\mu} \partial x^{\mu} /\left.\partial x^{i}\right|_{\Sigma}$ are

$$
\begin{aligned}
& \tilde{u}_{\tau}=-1, \\
& \widetilde{u}_{\theta}=\widetilde{u}_{\varphi}=0 .
\end{aligned}
$$

Assuming that the energy-momentum tensor $S_{i j}$ has the form $S_{i j}=\widetilde{\sigma} \tilde{u}_{i} \tilde{u}_{j}+\widetilde{\zeta}\left(\widetilde{g}_{i j}-\widetilde{u}_{i} \tilde{u}_{j}\right)$, from (43), we obtain

$$
\begin{aligned}
& S_{\tau}^{\tau}=-\tilde{\sigma}+2 \widetilde{\zeta}, \\
& S_{\theta}^{\theta}=S_{\varphi}^{\varphi}=\widetilde{\zeta} .
\end{aligned}
$$

From Israel conditions (45) written as

$$
\left[K_{j}^{i}\right]=-8 \pi G\left(S_{j}^{i}-\frac{1}{2} \delta_{j}^{i} S\right)
$$

we have

$$
\begin{aligned}
& {\left[K_{\tau}^{\tau}\right]=4 \pi G \widetilde{\sigma}} \\
& {\left[K_{\theta}^{\theta}\right]=\left[K_{\varphi}^{\varphi}\right]=-8 \pi G\left(\frac{\tilde{\sigma}}{2}-\widetilde{\zeta}\right) .}
\end{aligned}
$$

In the case of metric (3) with the function $f$ independent of $t$ from (40) one obtains

$$
\begin{aligned}
& K_{\tau}^{ \pm \tau}=\frac{1}{\dot{R}} \frac{d}{d \tau} \sqrt{f^{ \pm}(R)+\dot{R}^{2}}, \\
& K_{\theta}^{ \pm \theta}=K_{\varphi}^{ \pm \varphi}=\frac{1}{R} \sqrt{f^{ \pm}(R)+\dot{R}^{2}} .
\end{aligned}
$$

Israel conditions take a simple form in the case $\widetilde{\zeta}=0$. From the Israel conditions it follows that

$$
\begin{aligned}
\frac{1}{\dot{R}} \frac{d}{d \tau}\left[\sqrt{\dot{R}^{2}+f^{+}(R)}-\sqrt{\dot{R}^{2}+f^{-}(R)}\right] & =4 \pi \widetilde{\sigma}, \\
\frac{1}{R}\left[\sqrt{\dot{R}^{2}+f^{+}(R)}-\sqrt{\dot{R}^{2}+f^{-}(R)}\right] & =-4 \pi \widetilde{\sigma} .
\end{aligned}
$$

Solving this system of equations, we find that $R \widetilde{\sigma}=$ const and

$$
R\left(\sqrt{\dot{R}^{2}+f^{+}(R)}-\sqrt{\dot{R}^{2}+f^{-}(R)}\right)=M=\text { const. }
$$

Relation (55) can be rewritten as (cf.[8])

$$
\dot{R}^{2}+f^{ \pm}(R)-\frac{1}{M^{2}}\left[m_{+}-m_{-} \mp \frac{M^{2}}{2 R}\right]^{2}=0 .
$$

\section{Conclusions}

Geometry and connection between the two coordinate systems used to study dynamics of thin shells, KodamaSchwarzschild coordinates and Gaussian normal coordinate system, were studied. Transformation between the coordinate systems is studied and explicitly constructed for the case of Kodama-Schwarzschild metric independent of time. Extrinsic curvatures of the surface swept by the shell in the ambient space are calculated for a general time-dependent metric in both Kodama-Schwarzschild and normal Gaussian parametrizations and are shown to give the same result. Application to the Israel junction conditions is discussed. 


\section{Competing Interests}

The author declares that there is no conflict of interests regarding the publication of this paper.

\section{Acknowledgments}

This work was partially supported by the Ministry of Science and Education of Russian Federation under Project 01201255504.

\section{References}

[1] R. Brout, S. Massar, R. Parentani, and P. Spindel, "A primer for black hole quantum physics," Physics Reports, vol. 260, no. 6, pp. 329-446, 1995.

[2] A. Paranjape and T. Padmanabhan, "Radiation from collapsing shells, semiclassical backreaction, and black hole formation," Physical Review D, vol. 80, no. 4, Article ID 044011, 2009.

[3] W. Israel, "Singular hypersurfaces and thin shells in general relativity," Il Nuovo Cimento B Series, vol. 44, no. 1, pp. 1-14, 1966, Erratum in Il Nuovo Cimento B Series, vol. 48, p. 463, 1967.

[4] E. Poisson, A Relativist Toolkit, Cambridge University Press, Cambridge, UK, 2004.

[5] J. Ipser and P. Sikivie, "Gravitationally repulsive domain wall," Physical Review. D. Particles and Fields. Third Series, vol. 30, no. 4, pp. 712-719, 1984.

[6] V. A. Berezin, V. A. Kuzmin, and I. I. Tkachev, "Dynamics of bubbles in general relativity," Physical Review. D. Particles and Fields. Third Series, vol. 36, no. 10, pp. 2919-2944, 1987.

[7] S. K. Blau, E. I. Guendelman, and A. H. Guth, "Dynamics of false-vacuum bubbles," Physical Review D, vol. 35, no. 6, pp. 1747-1766, 1987.

[8] B. D. Chowdhury, "Problems with tunneling of thin shells from black holes," Pramana, vol. 70, no. 1, pp. 3-26, 2008.

[9] V. D. Gladush, "On the variational principle for dust shells in general relativity," Journal of Mathematical Physics, vol. 42, no. 6, pp. 2590-2610, 2001.

[10] P. Kraus and F. Wilczek, "Self-interaction correction to black hole radiance," Nuclear Physics B, vol. 433, no. 2, pp. 403-420, 1995.

[11] H. Kodama, "Conserved energy flux for the spherically symmetric system and the backreaction problem in the black hole evaporation," Progress of Theoretical Physics, vol. 63, no. 4, pp. 1217-1228, 1980.

[12] S. A. Hayward, "General laws of black-hole dynamics," Physical Review. D. Third Series, vol. 49, no. 12, pp. 6467-6474, 1994.

[13] S. A. Hayward, "Gravitational energy in spherical symmetry," Physical Review D, vol. 53, no. 4, pp. 1938-1949, 1996.

[14] S. A. Hayward, "Quasi-local gravitational energy," Physical Review D, vol. 49, no. 2, p. 831, 1994.

[15] G. Abreu and M. Visser, "Kodama time: geometrically preferred foliations of spherically symmetric spacetimes," Physical Review $D$, vol. 82, no. 4, Article ID 044027, 2010.

[16] J. Smoller and B. Temple, "Shock-wave solutions of the Einstein equations: the Oppenheimer-Snyder model of gravitational collapse extended to the case of non-zero pressure," Archive for Rational Mechanics and Analysis, vol. 128, no. 3, pp. 249-297, 1994.
[17] V. Faraoni, "Cosmological apparent and trapping horizons," Physical Review D, vol. 84, no. 2, Article ID 024003, 15 pages, 2011.

[18] C. W. Misner and D. H. Sharp, "Relativistic equations for adiabatic, spherically symmetric gravitational collapse," Physical Review, vol. 136, no. 2, pp. B571-B576, 1964.

[19] W. C. Hernandez Jr. and C. W. Misner, "Observer time as a coordinate in relativistic spherical hydrodynamics," The Astrophysical Journal, vol. 143, p. 452, 1966. 

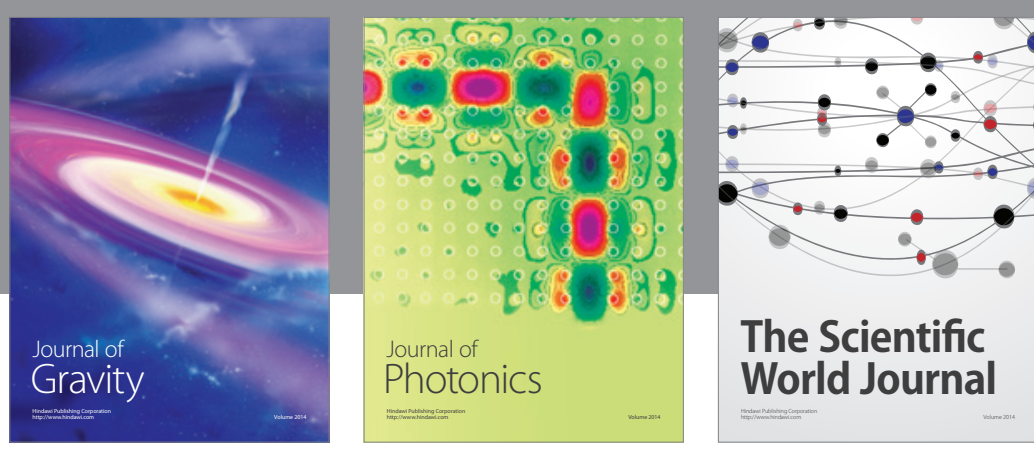

The Scientific World Journal
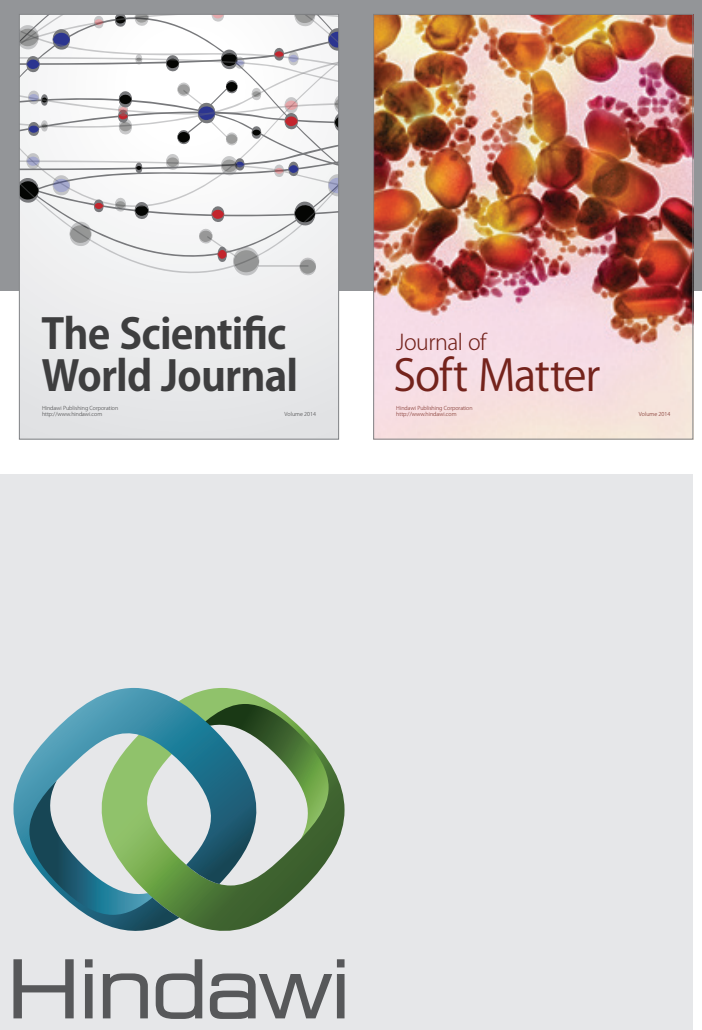

Submit your manuscripts at

http://www.hindawi.com

nternational Journal of

Statistical Mechanics
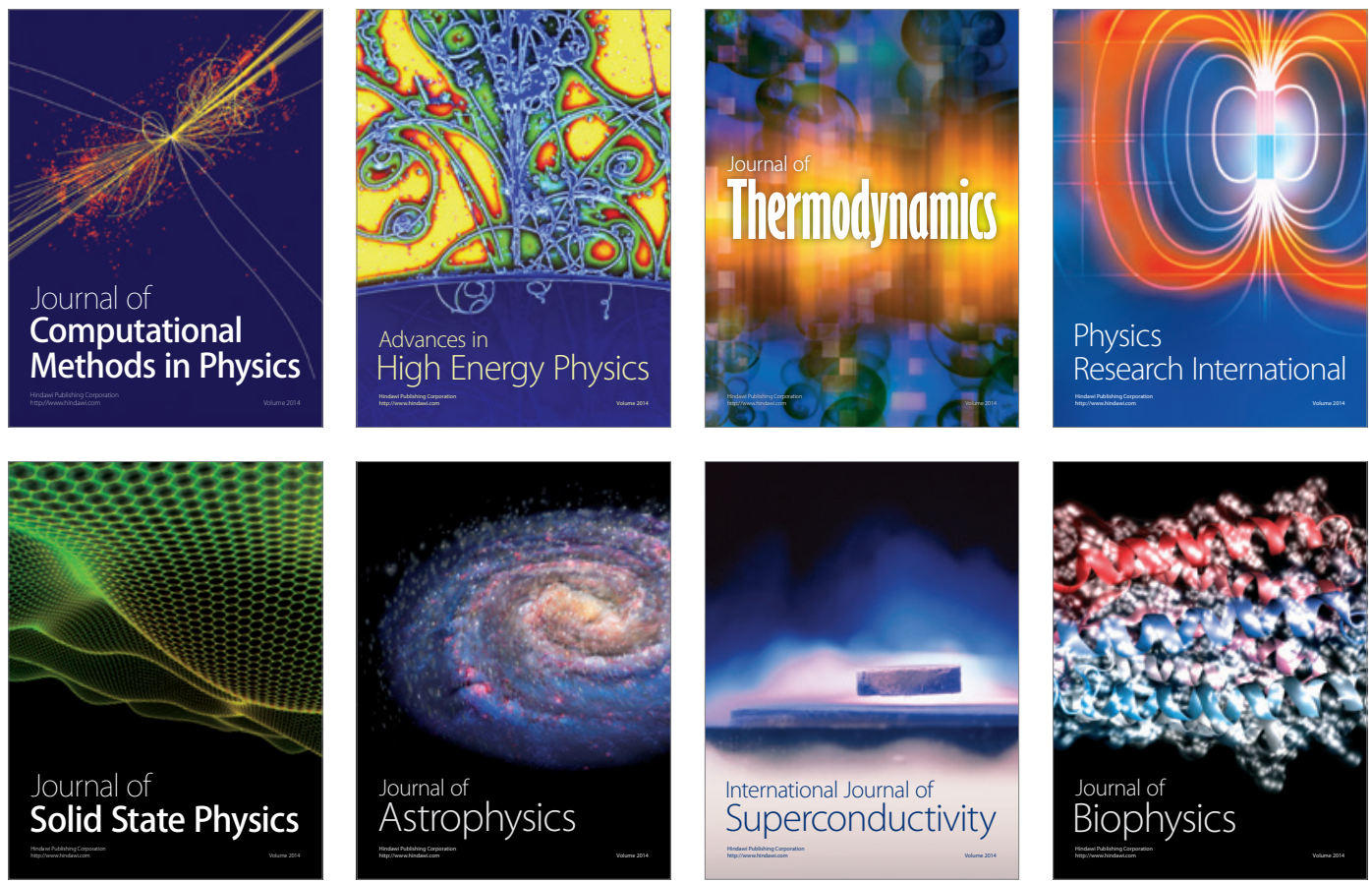
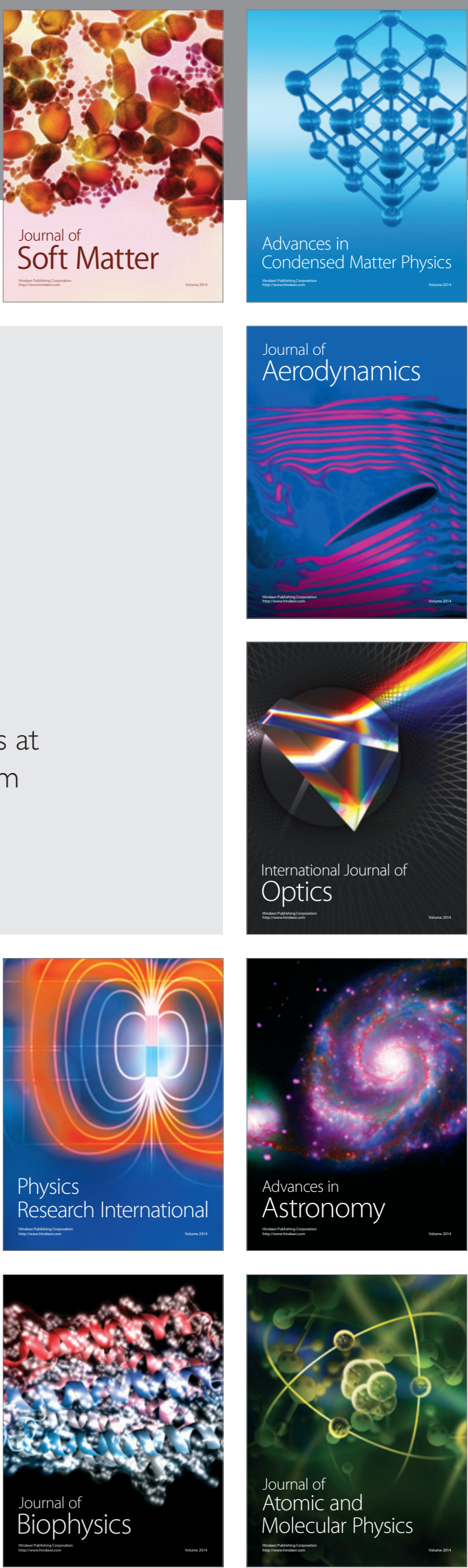